\title{
EFFECT OF GNRH ANTAGONISTS ON CLINICAL PREGNANCY RATES IN CONTROLLED OVARIAN STIMULATION AND INTRAUTERINE INSEMINATION
}

\author{
Rajat Kumar Ray ${ }^{1}$, Sunita Samal2
}

1Director, Ray Hospital and Test Tube Baby Centre, Rourkela.

${ }^{2}$ Consultant, Ray Hospital and Test Tube Baby Centre, Rourkela.

ABSTRACT

\section{BACKGROUND}

Controlled Ovarian Stimulation (COS) with Intrauterine Insemination (IUI) is widely used in the treatment of unexplained infertility, mild and moderate endometriosis or male factor infertility to achieve a higher pregnancy rate. Several studies have reported conflicting results regarding the outcome of COS-IUI cycles using GnRH antagonists in terms of clinical pregnancy rate.

The aim of the study is to study whether use of GnRH antagonist in IUI cycles where HMG was used for ovulation induction improved clinical pregnancy rates.

\section{MATERIALS AND METHODS}

This was a prospective randomised controlled trial study of 316 couples conducted at Ray Hospital and Test Tube Baby Centre from October 2013 to September 2015. The patients in group A received HMG and GnRH antagonist for COH, while those in group B received only HMG. Clinical pregnancy rates were compared.

Study Design- Randomised Controlled Trial.

Statistical Analysis- Statistical analyses were performed using the SPSS version 17.0 program (SPSS Inc., Chicago, IL, USA) for Windows. Student's t-test was used for comparing continuous variables that showed normal distribution, while Mann-Whitney U test was used for variables that did not follow a normal distribution.

\section{RESULTS}

Clinical pregnancy rate was $15.6 \%$ in GnRH antagonist group and $13.6 \%$ in the control group (P $=0.78)$.

\section{CONCLUSION}

The present study revealed no significant difference when GnRH antagonist is added to gonadotropin-stimulated IUI cycles in terms of clinical pregnancy rate.

\section{KEYWORDS}

Controlled Ovarian Stimulation, Intrauterine Insemination, GnRH Antagonist, Human Menopausal Gonadotropin, Clinical Pregnancy Rate.

HOW TO CITE THIS ARTICLE: Ray RK, Samal S. Effect of GnRH antagonists on clinical pregnancy rates in controlled ovarian stimulation and intrauterine insemination. J. Evolution Med. Dent. Sci. 2017;6(55):4150-4153, DOI: 10.14260/Jemds/2017/899.

\section{BACKGROUND}

Intrauterine Insemination (IUI) is widely used as an infertility treatment modality. Controlled Ovarian Stimulation (COS) with Intrauterine Insemination (IUI) is widely used in the treatment of unexplained infertility, mild and moderate endometriosis or male factor infertility to achieve a higher pregnancy rate. The underlying mechanism is based on the increase in the number of available ova and spermatozoa at the site of fertilisation. The target is to achieve two to three dominant follicles followed by a perfectly timed insemination. ${ }^{(1,2)}$ To increase the chances of success in terms of pregnancy rate in COS-IUI cycles, various therapeutic approaches have been tried by various researchers. These include- different ovarian stimulation protocols, double insemination and prevention of premature LH surge.

Financial or Other, Competing Interest: None.

Submission 19-06-2017, Peer Review 01-07-2017,

Acceptance 03-07-2017, Published 10-07-2017.

Corresponding Author:

Dr. Rajat Kumar Ray,

Director, Ray Hospital and Test Tube Baby Centre,

N-18, Civil Township, Rourkela-769004, India.

E-mail: rajatkuray@gmail.com

DOI: $10.14260 /$ jemds $/ 2017 / 899$
According to the Cochrane review on ovarian stimulation protocols for IUI in the women with subfertility, use of gonadotropins for COS in IUI results in higher pregnancy rate than clomiphene citrate stimulated cycles.(3) A recent metaanalysis clearly indicated that double insemination does not result in higher clinical pregnancy rate compared with single IUI in couples with unexplained infertility.(4) Double insemination has been suggested by researchers because of the hypothesis that capacitated sperms in the inseminate are active for only 2-3 hrs., so they may not be able to backup ovulation, which takes place in between the next 20 and 24 hrs. However, it appears that precise timing of insemination in relation to ovulation, so as to enable active sperms to reach and fertilise the oocyte should obviate the need for double insemination.

Although, low-dose protocols with gonadotropins results in a higher pregnancy rate, recruitment of multiple follicles rapidly increases the serum $\mathrm{E} 2$ levels, which may increase the risk of a premature LH surge (PL). Approximately, 20\% of COS/IUI cycles have been shown to undergo PL.(5) PL has been reported to be detrimental to oocyte quality, fertilisation and embryo implantation. LH surge can be effectively prevented by administering a GonadotropinReleasing Hormone (GnRH) agonist or GnRH antagonist. $(6,7)$ $\mathrm{GnRH}$ analogues lead to the desensitisation of pituitary $\mathrm{GnRH}$ 
receptors and block endogenous LH increase. It needs 2-3 weeks of desensitisation time, also increases the amount of gonadotropins used, the risk of ovarian hyperstimulation syndrome and the duration of treatment. It has got antioestrogenic side effects such as hot flushes, headaches, vaginal dryness and bleeding. These drawbacks have resulted in the recent removal of IUI treatment protocols including $\mathrm{GnRH}$ agonists. On the other hand, GnRH antagonists bind to GnRH receptors with high affinity. It acts rapidly resulting in preventing the release of endogenous gonadotropin within 2$4 \mathrm{hrs}$. Its advantage over GnRH analogues are absence of the first flare-up effect, reduced risk of oestrogen deficiency syndrome, sufficient LH blockage in a short duration of time, dose-dependent effect, fast withdrawal from antagonist effects, reduced treatment duration and the total dose of gonadotropin and a low risk of high order multiple pregnancies when standard strict criteria are applied for cycle cancellation.

Several studies have reported conflicting results regarding the outcome of COS-IUI cycles using GnRH antagonists in terms of clinical pregnancy rate. So, we decided to study whether use of GnRH antagonist in IUI cycles where HMG was used for ovulation induction improved clinical pregnancy rates.

\section{MATERIALS AND METHODS}

This was a prospective, randomised-controlled trial study conducted at Ray Hospital and Test Tube Baby Centre from October 2013 to September 2015. 316 couples with unexplained subfertility meeting the inclusion criteria were included in the study. Written informed consent was obtained from all couples before randomisation. The study was approved by the local ethics committee.

\section{Inclusion Criteria was-}

- Unexplained infertility.

- Minimal or mild endometriosis.

- At least 5 million/mL sperm count and 5\% normal morphology according to strict Kruger's criteria.

- Bilateral tubal patency and normal uterine cavity on HSG or diagnostic hysteroscopy and laparoscopy.

- Regular menstrual cycle.

- Basal FSH $\leq 10 \mathrm{IU} / \mathrm{L}$.

- Previous max. 2 cycles of IUI with CC.

- Normal levels of TSH and prolactin.

\section{Patients with Following Conditions were Excluded from} the Study-

- Age $\geq 38$ years.

- PCOS.

- Unilateral tubal patency or hydrosalpinx.

- Endometriosis stage III/IV.

The Treatment Cycle was Cancelled in the Following Circumstances-

- No follicle $\geq 14 \mathrm{~mm}$.

- $\geq 3$ follicles of $\geq 16 \mathrm{~mm}$ on the day hCG.

- Endometrial thickness $\leq 6 \mathrm{~mm}$ on the day of IUI.

- Premature luteinisation.

- Progesterone level $>1.7 \mathrm{ng} / \mathrm{dL}$ on the day of hCG.

- LH level $>10 \mathrm{mIU} / \mathrm{mL}$ on the day of hCG.
The patients were randomly divided into two groups, i.e. study group or Group A and control group or Group B using computer generated randomisation software. The patients in Group A received HMG and GnRH antagonist for $\mathrm{COH}$, while those in group B received only HMG. In both groups, ovarian stimulation was started on the second day of the menstrual cycle after performing a basal scan to rule out any cysts in ovary and also to confirm endometrial thickness $<5 \mathrm{~mm}$.

We took into consideration the age of women, Body Mass Index (BMI) and history of previous ovarian response to stimulation with clomiphene to decide the starting dose of HMG. Most of the patients were started with $75 \mathrm{IU} / \mathrm{mL}$ of HMG. Repeat transvaginal scanning was done on $7^{\text {th }}$ or $8^{\text {th }}$ day and the subsequent doses were adjusted according to the follicular size. In Group A patients, when the leading follicle reached a size of $14 \mathrm{~mm}$, GnRH antagonist Cetrorelix was added and continued along with HMG daily till the day of hCG. In both groups, when at least one follicle reached a diameter of $18 \mathrm{~mm}$, hCG $5000 \mathrm{IU}$ was given intramuscularly to trigger the ovulation.

Semen was prepared with double density gradient technique. Single intrauterine insemination was performed 36-38 hrs. after hCG injection. Luteal support in both groups was given with micronized vaginal progesterone $400 \mathrm{mg}$ twice daily. Transvaginal sonography was performed after 20-25 days of IUI to confirm presence of gestational sac. Once intrauterine gestational sac was seen, subsequent TVS was done at appropriate time to confirm viability and rule out multiple pregnancy. Luteal support was continued till 10 weeks post-IUI. In both groups, every couple was subjected to only one cycle of treatment.

The following outcome measures were compared between groups A and B- (a) Primary outcome measureclinical pregnancy rate and (b) Secondary outcome measures(1) Pregnancy loss rate, (2) Multiple pregnancy rate, (3) Ovarian hyper-response rate.

Clinical pregnancy was defined as- Pregnancy diagnosed by ultrasound visualisation of at least one gestational sac with or without cardiac activity. Pregnancy loss was defined as- abortion (up to 12 weeks) and ectopic pregnancy. Multiple pregnancies were defined as- Ultrasound visualisation of more than one gestational sac with or without cardiac activity. Ovarian hyper-response was defined as $\geq 4$ follicles $>14 \mathrm{~mm}$ on the day of hCG.

\section{Statistical Analysis}

Statistical analyses were performed using the SPSS version 17.0 program (SPSS Inc. Chicago, IL, USA) for Windows. Student's t-test was used for comparing continuous variables that showed normal distribution, while Mann-Whitney U test was used for variables that did not follow a normal distribution. To compare qualitative data, Chi-square test and Fisher's exact test were used. $\mathrm{P}<0.05$ was considered statistically significant.

\section{RESULTS}

In our study, 316 eligible couples were randomised into study and control groups. 10 patients in study group and 7 patients in control group were lost to follow up. 2 patients in study group did not have timely TVS. 5 cases in study group and 4 cases in control group did not have adequate response. So, 288 patients were included in the data analysis, which included 141 cases in study group and 147 cases in control group. 
The characteristics of IUI treatment cycles were also comparable in patients of two groups (Table 1). The differences in mean age, BMI and duration of infertility between groups A and B were not statistically significant. The total dose of HMG was significantly higher in GnRH antagonist group compared with control group (984.76 \pm 342.38 vs. $756.84 \pm 312.41)(\mathrm{P}=0.001)$.

The results of study are shown in Table 2. Clinical pregnancy rate was $15.6 \%$ (22) in GnRH antagonist group and $13.6 \%(20)$ the control group $(\mathrm{P}=0.78)$. Spontaneous pregnancy loss was observed in $16.3 \%$ (23) cases in $\mathrm{GnRH}$ antagonist group and $14.3 \%$ (21) cases in the control group $(\mathrm{P}=0.68)$. Similarly, multiple pregnancy rates were similar in two groups, $13.6 \%$ (3) in GnRH antagonist group and 15.0\% (3) in control group ( $\mathrm{P}=0.42)$. Incidence of ovarian hyperresponse was significantly higher in control group, $16.3 \%$ (24) when compared to GnRH antagonist group, 8.5\% (12) (P $=0.016)$.

\begin{tabular}{|c|c|c|c|}
\hline Variables & Study Group & Control Group & p-value \\
\hline $\begin{array}{c}\text { Duration of } \\
\text { infertility (in } \\
\text { years) }\end{array}$ & $3.0(2-5)$ & $3.5(2-5.5)$ & 0.601 \\
\hline Age (in years) & $29.5 \pm 4.58$ & $30.1 \pm 3.62$ & 0.12 \\
\hline BMI (kg/m²) & $25.5 \pm 3.81$ & $25.9 \pm 4.29$ & 0.825 \\
\hline $\begin{array}{c}\text { Total dose of } \\
\text { HMG (IU) }\end{array}$ & $984.76 \pm 342.38$ & $756.84 \pm 312.41$ & 0.001 \\
\hline \multicolumn{3}{|c|}{ Table 1. Characteristics of Treatment Cycles } \\
\hline
\end{tabular}

\begin{tabular}{|c|c|c|c|}
\hline Outcome Measures & Study Group & Control Group & p-value \\
\hline $\mathbf{n}$ & $\mathbf{1 4 1}$ & $\mathbf{1 4 7}$ & \\
\hline $\begin{array}{c}\text { Clinical pregnancy } \\
\text { rate }\end{array}$ & $15.6 \%(22)$ & $13.6 \%(20)$ & 0.78 \\
\hline Pregnancy loss rate & $16.3 \%(23)$ & $14.3 \%(21)$ & 0.68 \\
\hline $\begin{array}{c}\text { Multiple pregnancy } \\
\text { rate }\end{array}$ & $13.6 \%(3 / 22)$ & $15.0 \%(3 / 20)$ & 0.42 \\
\hline $\begin{array}{c}\text { Ovarian hyper- } \\
\text { response rate }\end{array}$ & $8.5 \%(12)$ & $16.3 \%(24)$ & 0.016 \\
\hline \multicolumn{3}{|c|}{ Table 2. Primary and Secondary Outcomes } \\
\hline \multicolumn{4}{|c|}{}
\end{tabular}

\section{DISCUSSION}

Our study aimed to explore the efficacy of GnRH antagonist when used as an adjuvant to COS-IUI cycles. Several studies have shown that premature LH surges occur in a significant percentage of COS cycles interfering with the optimal timing of the insemination or even resulting in cycle cancelation. Premature LH has also been linked with lower pregnancy rates compared with cycles without a premature LH surge.(8) The role of GnRH antagonist in prevention of premature $\mathrm{LH}$ surge is well-established.(9)

Our study could not confirm the effect of GnRH antagonist on improving clinical pregnancy rate in COS-IUI cycles.

Studies have shown that premature LH surge did not start before follicle size of $14-15 \mathrm{~mm}$ in majority of cases.(10) Hence, we have taken follicle size $>14 \mathrm{~mm}$ as the cut-off limit to start GnRH antagonist. In control group, premature $\mathrm{LH}$ surge started in four cycles. To avoid a hypothetical effect of antagonist on function of corpus luteum, we routinely gave luteal phase support to all women in both groups.

Both groups were comparable in demographic characteristics regarding age, BMI and duration of infertility, which rule out the selection bias. There was no significant difference in most of the treatment variables between the two groups, except the total dose of gonadotropin, which was significantly higher in GnRH antagonist group (984 vs. 756).

The growth and maturation of a dominant follicle is not affected by sudden $\mathrm{LH}$ withdrawal induced by $\mathrm{GnRH}$ antagonist, but there is arrest in growth of intermediate size follicles.(10) So, when GnRH antagonist is added to suppress $\mathrm{LH}$, the smaller follicles having higher LH threshold get atretic and there are ultimately lesser follicles achieving dominance. This explains the reduction in ovarian hyper-response rate with the addition of GnRH antagonist. In our study, there is significant reduction in ovarian hyper-response rate with the addition of GnRH antagonist (8.5\% vs. $16.3 \%$ ). There was no case of Ovarian Hyperstimulation Syndrome (OHSS) in our study. More number of mature follicles also increases the risk of multiple gestations. The difference between multiple pregnancy rates were not statistically significant in study and control groups (13.6\% vs. $15.0 \%)$. There was no case of highorder multiple pregnancies in our study. No increase in multiple pregnancies or OHSS is attributable to the strict cancellation criteria in our study.

The difference between pregnancy loss rates were not statistically significant in study and control groups $(16.3 \%$ vs. $14.3 \%)$. We also did not find a statistically significant difference in clinical pregnancy rates between study and control groups ( $15.6 \%$ vs. $13.6 \%$ ).

There is no consensus regarding the use of GnRH antagonists during $\mathrm{COH}+\mathrm{IUI}$ cycles in the literature. Kosmas et al(11) performed a meta-analysis in 2008 , which consisted of six trials with 1,069 subjects and reported significantly higher clinical pregnancy rate per cycle in favour of treatment with GnRH-antagonist. In the last Cochrane systematic review regarding optimal stimulation protocols for IUI, five RCTs up to 2007 were included and it was concluded that adding a GnRH antagonist does not increase pregnancy rate (OR 1.5, 95\%, CI-0.83-2.8). A multicentre randomised study by Crosignani et al in 2007 failed to prove that the use of GnRH antagonists is beneficial in IUI cycles.(12) In another study in 2009, Martinez-Salazar et al concluded that while the universal use of GnRH antagonists in COS-IUI cycles does not increase pregnancy rates, it may benefit a specific subset of patients who have premature luteinisation or high progesterone concentrations in a previous failed IUI.(13) Another large double-blinded, placebo-controlled, multicenter trial by Cantineau et al in 2011 proved that $\mathrm{GnRH}$ antagonist addition does not increase livebirth rate in gonadotropin-stimulated IUI cycles.(14) The results of our study are in line with the available evidence.

The use of GnRH antagonists has also been shown to be effective in manipulating follicular development, so that the insemination process can be suspended on weekends without an apparent decrease in pregnancy rates.(15)

\section{CONCLUSION}

The present study revealed no significant difference when GnRH antagonist is added to gonadotropin-stimulated IUI cycles in terms of clinical pregnancy rate. There is also no significant reduction of pregnancy loss rate or multiple pregnancy rates. However, there is a significant difference in the ovarian hyper-response rate. However, GnRH antagonist treatment could allow flexible timing of hCG injection and insemination, thereby avoiding IUI during weekends. Larger randomised controlled trials are required to reach useful 
conclusions about the use of GnRH antagonists in COS-IUI cycles.

\section{REFERENCES}

[1] Verhulst SM, Cohlen BJ, Hughes E, et al. Intrauterine insemination for unexplained subfertility. Cochrane Database Syst Rev 2006;4:CD001838.

[2] Bensdorp AJ, Cohlen BJ, Heineman MJ, et al. Intrauterine insemination for male subfertility. Cochrane Database Syst Rev 2007;3:CD000360.

[3] Cantineau AE, Cohlen BJ, Heineman MJ. Ovarian stimulation protocols (anti-oestrogens, gonadotrophins with and without GnRH agonists/antagonists) for intrauterine insemination (IUI) in women with subfertility. Cochrane Database Syst Rev 2007;2:CD005356.

[4] Polyzos NP, Tzioras S, Mauri D, et al. Double versus single intrauterine insemination for unexplained infertility: a meta-analysis of randomized trials. Fertil Steril 2010;94(4):1261-6.

[5] Ragni G, Somigliana E, Vegetti W. Timing of intrauterine insemination: where are we? Fertil Steril 2004;82(1):25-6, 32-5.

[6] Lee TH, Lin YH, Seow KM, et al. Effectiveness of Cetrorelix for the prevention of premature luteinising hormone surge during controlled ovarian stimulation using letrozole and gonadotropins: a randomized trial. Fertil Steril 2008;90(1):113-20.

[7] Alisch A, Roiha K, Finas D, et al. Extreme suppression of LH within 3 hours after GnRH-antagonist administration in $\mathrm{COH}$ : results of pulsatility pattern analysis. Hum Reprod 2004;19(Suppl 1):i61.

[8] Cantineau AE, Cohlen BJ, Dutch IUI study group. The prevalence and influence of luteinizing hormone surges in stimulated cycles combined with intrauterine insemination during a prospective cohort study. Fertil Steril 2007;88(1):107-12.
[9] Lambalk CB, Leader A, Olivennes F, et al. Treatment with the GnRH antagonist Ganirelix prevents premature LH rises and luteinization in stimulated intrauterine insemination: results of a double-blind, placebo-controlled, multicentre trial. Hum Reprod 2006;21(3):632-9.

[10] Mochtar MH, Dutch Ganirelix Study Group. The effect of an individualized GnRH antagonist protocol on folliculogenesis in IVF/ICSI. Hum Reprod 2004;19(8):1713-8.

[11] Kosmas IP, Tatsioni A, Kolibianakis EM, et al. Effects and clinical significance of GnRH antagonist administration for IUI timing in FSH superovulated cycles: a meta-analysis. Fertil Steril 2008;90(2):36772.

[12] Crosignani PG, Somigliana E, Intrauterine Insemination Study Group. Effect of GnRH antagonists in FSH mildly stimulated intrauterine insemination cycles: a multicentre randomized trial. Hum Reprod 2007;22(2):500-5.

[13] Martinez-Salazar J, Cerrillo M, Quea G, et al. GnRH antagonist Ganirelix prevents premature luteinization in IUI cycles: rationale for its use. Reprod Biomed Online 2009;19(2):156-61.

[14] Cantineau AE, Cohlen BJ, Klip H, et al. The addition of GnRH antagonists in intrauterine insemination cycles with mild ovarian hyperstimulation does not increase live birth rates - a randomized, double-blinded, placebo-controlled trial. Hum Reprod 2011;26(5):1104-11.

[15] Checa MA, Prat M, Robles A, et al. Use of gonadotropin releasing hormone antagonists to overcome the drawbacks of intrauterine insemination on weekends. Fertil Steril 2006;85(3):573-7. 